\title{
EFFECT OF GEOMETRY ON PERFORMANCE OF INTERLACER
}

\author{
Qiu Hua*, Fu Yu ye, Ge Mingqiao
}

Key Laboratory of Eco-textiles, Ministry of Education, Jiangnan University, Jiangsu, China e-mail: qiuhua@jiangnan.edu.cn

\begin{abstract}
:
Interlacer is the key part of interlacing technology that is adopted to improve the cohesion between loose multifilaments. Aimed at finding the interlacers with better performance, the present research designed five interlacers that can be classified into round type and cornered type. These five interlacers are different in cross-sectional shapes of yarn channel but are the same in the cross-sectional area. The evaluation of the performance of the interlacer includes the number and the strength of the tangles of the interlaced yarn it produces. Experiments are carried out at various supplied air pressures, yarn speeds and feed ratios. It was found that the interlacer with round cross-sectional shape of yarn channel is capable of producing an interlaced yarn with a large number of tangles and the cornered cross-sectional shape is effective in improving the strength of tangles. Among these five interlacers, the interlacer with an elliptical or an inverse-triangular shape has the best processing performance.
\end{abstract}

\section{Keywords:}

Interlacer, Interlaced yarn, Shape of yarn channel, Number of tangles, Strength of tangles

\section{Introduction}

Interlacing is an effective technique to impart cohesive force to multi-filament yarn at relatively lower costs compared with sizing and twisting [1]. The interlacer plays an important role in the interlacing process. A simple interlacer is composed of an air jet nozzle, a yarn channel and two yarn guides. By blowing compressed air on a yarn running through the yarn channel, filaments tangle with each other to form interlaced yarn with alternate tangling and opening parts. The first patent for interlacer was introduced by DuPont in 1961 [2]. From then on, many useful experiments have been carried out. Also, lemoto et al. investigated the effects of supplied air pressure $p$, airflow rate $Q$, yarn speed $v$ and overfeed ratio $F$ on the number of tangles [3]. Miao investigated the effects of interlacing on characteristics of interlaced yarn and the relations among processing parameters and linear density, tenacity and abrasion resistance of interlaced yarn [4]. Further, lemoto investigated yarn positions on the crosssection of yarn channel with a still camera [5]. Many studies have been carried out to make clear some airflow patterns, such as static pressure on yarn channel [6] and dynamic pressure near yarn channel [7], by an experimental method [7], computation method [8] or by a combination of these two methods [9]. These studies focused on improving the performance of interlaced yarn and clarifying the mechanism of interlacing. In most of these researches, yarn channels of interlacers had a circular crosssection. Some patents and papers designed non-circular yarn channel shapes but there were no detailed comparisons [10]. Actually, cross-sectional shape of yarn channel decides the distribution of airflow and then the movement of filaments in yarn channel [11]. As a result, the performance of interlaced yarn will also be affected.
The main aim of this work is to find the relations between cross-sectional shape of yarn channel and the performance of interlacer. Five interlacers having variant cross-sectional shapes of yarn channel with the same cross-sectional area are designed. Here, the performance of an interlacer is evaluated from the number and strength of interlaced yarn it produces.

\section{Experiment}

The supply yarn for present research was 16.7 tex/48 filaments, a common industrial yarn.

Geometry of the interlacer is shown in Figure 1. The diameter of air jet nozzle is $1.4 \mathrm{~mm}$, and length of yarn channel is $14.7 \mathrm{~mm}$ [12].

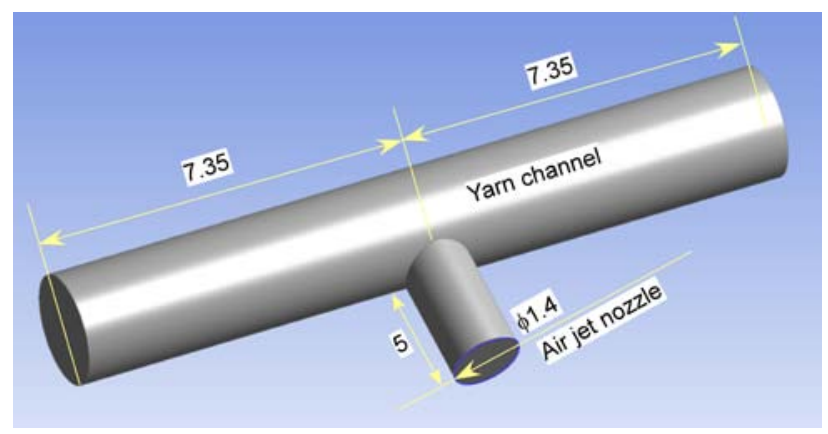

Figure 1. Interlacer (unit: $\mathrm{mm}$ ).

These five types of yarn channels have the same crosssectional area. In Figure 2, E1, E2 and E3 are interlacers with an elliptical yarn channel. T1 and T2 have a triangular 
one. These interlacers can be classified into two classes: yarn channel with round shape, E1, E2 and E3; yarn channel with cornered shape, T1 and T2. For the sake of brevity, the interlacers having elliptical cross-sectional shapes of yarn channel are referred to as round interlacers, and the others as the cornered interlacers.

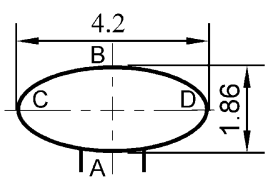

E1

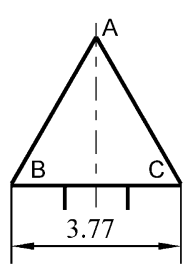

T1

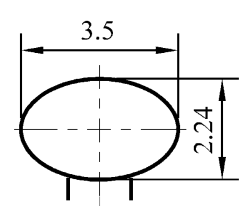

E2

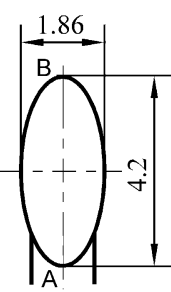

E3

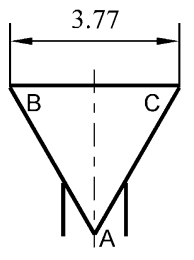

$\mathrm{T} 2$
Figure 2. CCross-sectional shapes of yarn channel (unit: $\mathrm{mm}$ ).

Experiments were carried out under the following parameters: supplied air pressure $p$ was regulated from 0.1 to $0.5 \mathrm{MPa}$ with a step of $0.1 \mathrm{MPa}$; yarn speed $v$ (linear surface velocity of the feed roller) was changed from 200 to $800 \mathrm{~m} / \mathrm{min}$ with a step of $200 \mathrm{~m} / \mathrm{min}$; feed ratio $F$ was changed from $1 \%$ to $5 \%$ with a step of $1 \%$; and initial yarn tension was fixed at $4.5 \mathrm{mN} /$ tex.

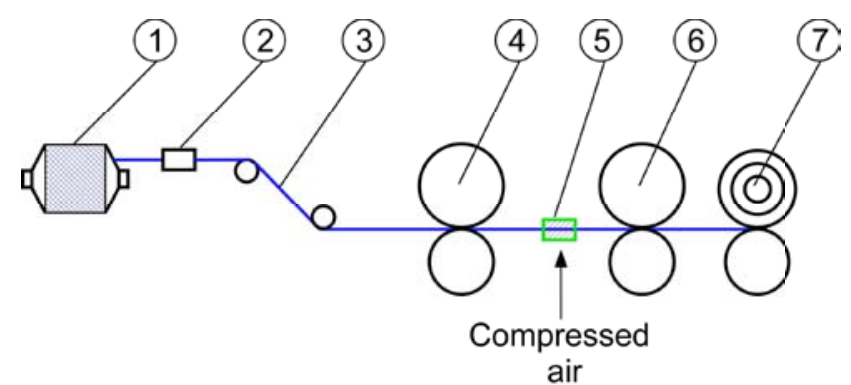

$\begin{array}{llll}\text { (1) Bobbin } & \text { (2) Tensioner } & \text { (3) Yarn } & \text { (4) Feed roller } \\ \text { (5) Interlacer } & \text { (6) Delivery roller } & \text { (7) Take-up roller }\end{array}$

Figure 3. Schematic model of experimental apparatus.

Figure 3 shows the schematic model of experimental apparatus. Yarn (3) is released from bobbin(1) and passes through tensioner(2) that can adjust an initial yarn tension. After a feed roller(4), the yarn is interlaced in an interlacer(5) under the action of compressed air. Then the interlaced yarn passes through a delivery roller(6) and is wounded on a takeup roller(7). The region between the feed roller and delivery roller is called a processing section.

For each interlaced yarn sample processed in a combination of experimental conditions of $p, v$ and $F$, three specimens were taken from different parts of the interlaced yarn package. The length of each specimen was $2 \mathrm{~m}$ measured at a tension of $2.97 \mathrm{mN} / \mathrm{tex}$. The measured length of interlaced yarn was decided at a tension of $2.97 \mathrm{mN} / \mathrm{tex}$.

$N$ is referred to as number of tangles that is defined as the tangling number in interlaced yarn per metre. The strength of tangles $S$ is defined as $N_{\mathrm{r}} / N \times 100$, where $N r$ is the remaining tangling number in interlaced yarn per metre after the action of the load of $135 \mathrm{mN} /$ tex for $3 \mathrm{~min}$. In general, larger $N$ is valuable. Too large $S$ is not desirable because tangling parts remaining in cloth become a fault, and small $S$ is not good either. Even though $S$ is large, an interlaced yarn with low $N$ is not good. In this research, performance of the interlaced yarn is evaluated by the mean values $N$ and $S$, respectively. And $S$ more than $95 \%$ is judged to be too large and $S$ less than $75 \%$, small.

\section{Results and Discussion}

\section{Comparison among E1, E2 and E3}

Figure 4 makes the comparison in $N$ among E1, E2 and E3 at $F=2 \%$ and $v=200,400$ and $600 \mathrm{~m} / \mathrm{min}$. In Figure 4 (a), there are only two plots at $p=0.5 \mathrm{MPa}$, because E1 and E3 produce the same $N$. In Figure 4 (c), there are only two plots at $p=$ $0.1 \mathrm{MPa}$, because E3 cannot produce interlaced yarn under this condition. With an increase in $p$, for interlacers E1, E2 and E3, $N$ increases first and then decreases, getting maximum at $p=0.3 \mathrm{MPa}$. Generally speaking, when $p$ is lower, filaments are more difficult to open and $N$ is smaller. With increasing $p$, filaments can be opened fully and $N$ becomes higher. However, compressed air with too higher $p$ will make filaments stay near the top of yarn channel and $N$ decreases.

Figure 5 shows the comparison in $N$ among $\mathrm{E} 1, \mathrm{E} 2$ and E3 at $p=0.4 \mathrm{MPa}$ and $F=2 \%$. From the figure, $N$ decreases monotonously with an increase in $v$. When yarn speed is higher, the time for filaments to be subjected to the compressed air becomes short and filaments are more difficult to open and tangle with each other.

Figure 6 shows the comparison in $N$ among E1, E2 and E3 at $p=0.4 \mathrm{MPa}$ and $v=200 \mathrm{~m} / \mathrm{min}$. The figure indicates that $N$ increases monotonously with $F$. Higher $F$ means lower yarn tension in the processing section and filaments are more easily opened by compressed air. However, increasing $F$ to improve $N$ is not advisable because the opening parts of the interlaced yarn produced at $F=5 \%$ have big loops as shown in Figure 7, which contains pictures of the interlaced yarn produced with E2 at $p=0.3 \mathrm{MPa}, v=200 \mathrm{~m} / \mathrm{min}$ and $F=2$ and $5 \%$, respectively.

From Figures 4-6, it can be seen that E2 can produce interlaced yarn with the largest $N$ and E3 produces the smallest $N$ among these three elliptical interlacers.

Table 1 shows the performance of E1, E2 and E3. As shown in Table 1, E1 and E2 can produce interlaced yarn under most of the processing parameters. When $F \geq 2 \%$, and at lower $p$ and higher $v$, E3 cannot produce interlaced yarn. The region 

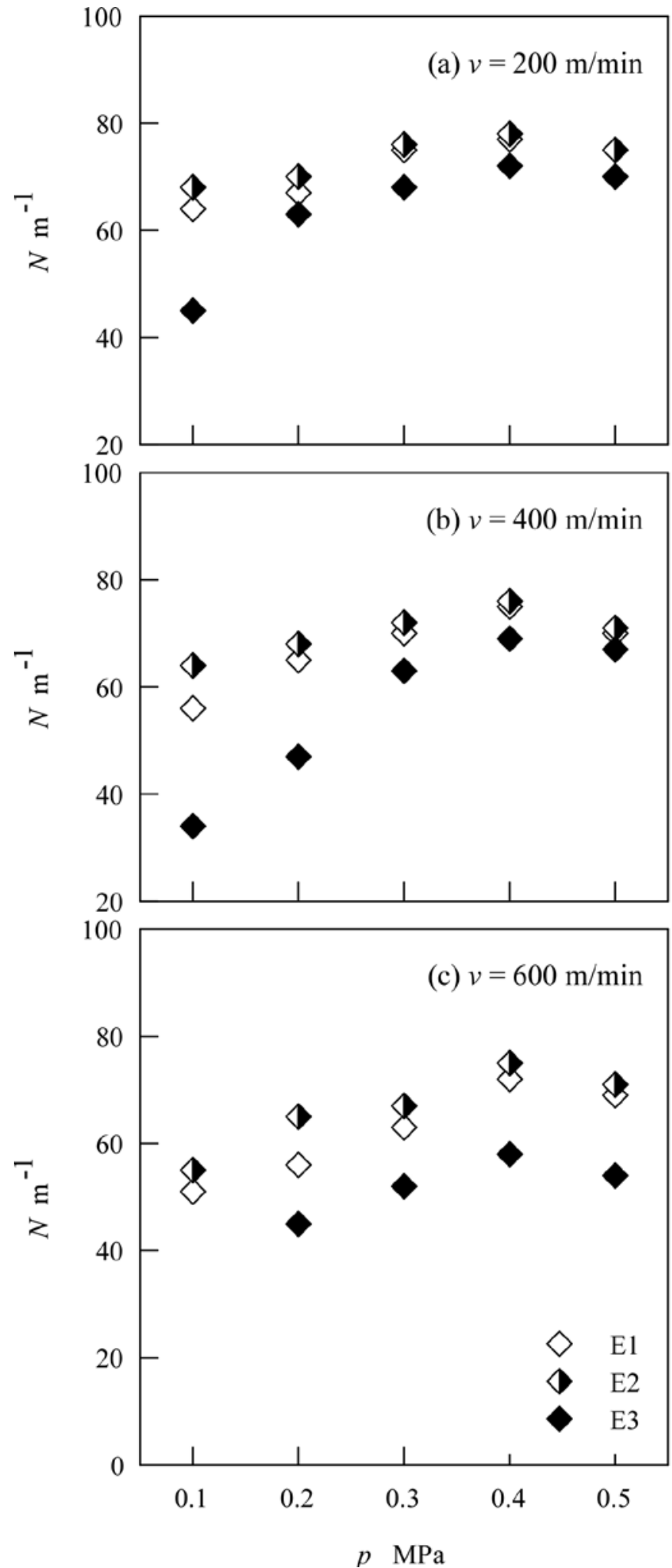

Figure 4. Comparison in $N$ among $\mathrm{E} 1, \mathrm{E} 2$ and $\mathrm{E} 3$ at $F=2 \%$.

where tangling parts of filaments are produced in yarn channel is referred as processing space. Among E1, E2 and E3, the difference between major and minor axes of E3 is distinct. As a result, the processing space of E3 is narrower compared with $\mathrm{E} 1$ and E2. When $v$ and $F$ are higher and $p$ is lower, filaments are difficult to be opened and are tangled with each other in such a narrow processing space. With processing going on, too many yarns stagnate between feed roller and delivery

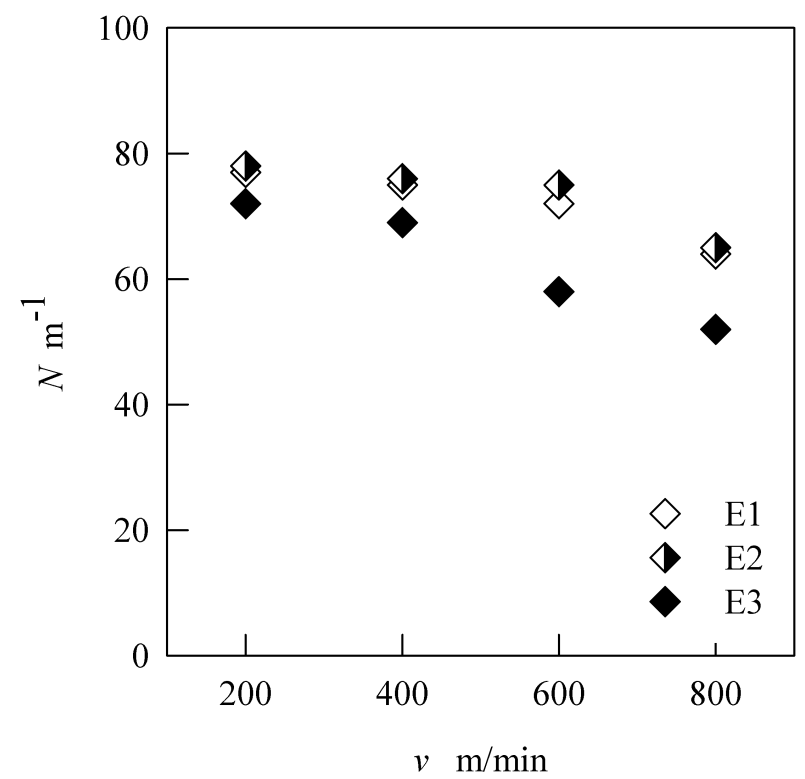

Figure 5. Comparison in N among E1, E2 and E3 at $p=0.4 \mathrm{MPa}$ and $F=2 \%$.

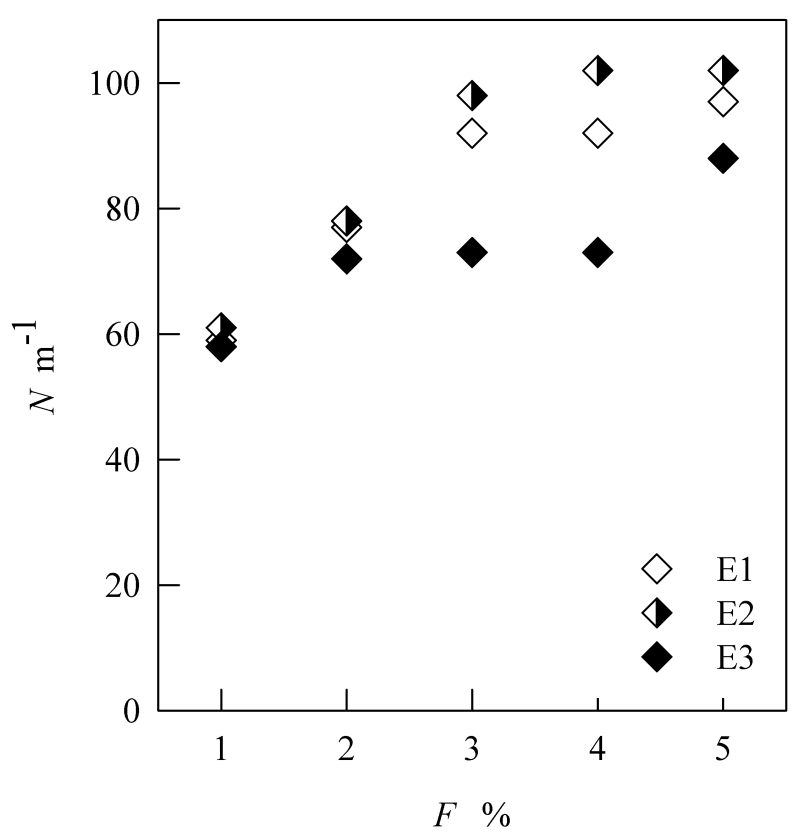

Figure 6. Comparison in $N$ among E1, E2 and E3 at $p=0.4 \mathrm{MPa}$ and $v=200 \mathrm{~m} / \mathrm{min}$.

$10 \mathrm{~mm}$

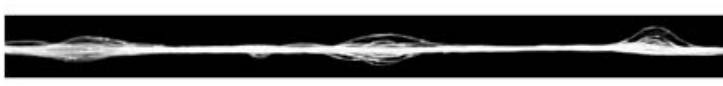

(a) $F=2 \%$

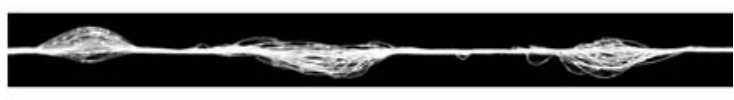

(b) $F=5 \%$

Figure 7. Picture of the interlaced yarn produced with $\mathrm{E} 2$ at $F=2 \%$ and $v=200 \mathrm{~m} / \mathrm{min}$. 
Table 1. Performance of E1, E2 and E3

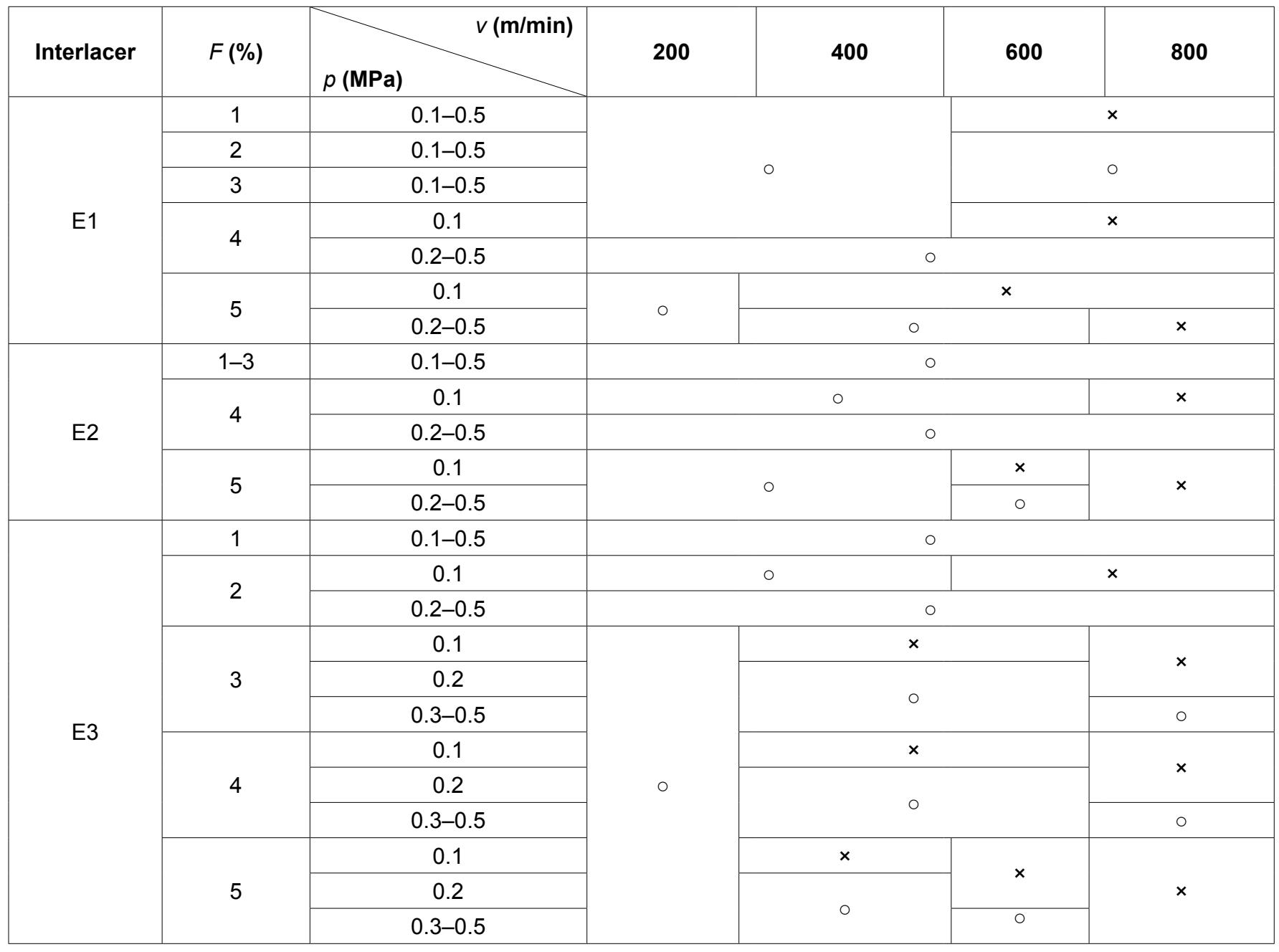

o indicates that yarn can be interlaced.

$x$ indicates that yarn cannot be interlaced.

roller and will be wounded by feed roller, because yarn cannot shrink. E2 can produce the interlaced yarn with larger $N$ than $\mathrm{E} 1$ under most of the experimental conditions. Compressed air issuing from air jet nozzle moves from position $A$ to $B$ as shown in Figure 1 with diffusion and then forms two vortexes. With the revolution of compressed air, filaments will be separated and then tangle with each other to form tangling parts. Because the length of major axis of E1 is longer than that of E2, filaments will stay at positions $C$ and $D$ for a longer time and it is difficult for them to tangle with each other.

Figure 8 shows the comparison in $S$ among E1, E2 and E3 at $F=2 \%$ and $v=200 \mathrm{~m} / \mathrm{min}$. From Figure 8 , E3 can produce interlaced yarn with the highest $S$. E1 and E2 produce almost the lowest $S$. However, for each interlacer, $S$ changes a little with $p$ increasing. For $\mathrm{E} 3$, the length difference of major and minor axes is largest, so the rotating speed difference of filaments is the largest and they can tangle with each other tightly.

Among these three elliptical interlacers, performance of E2 is the best, producing interlaced yarn with the largest $N$ and highest $S$.

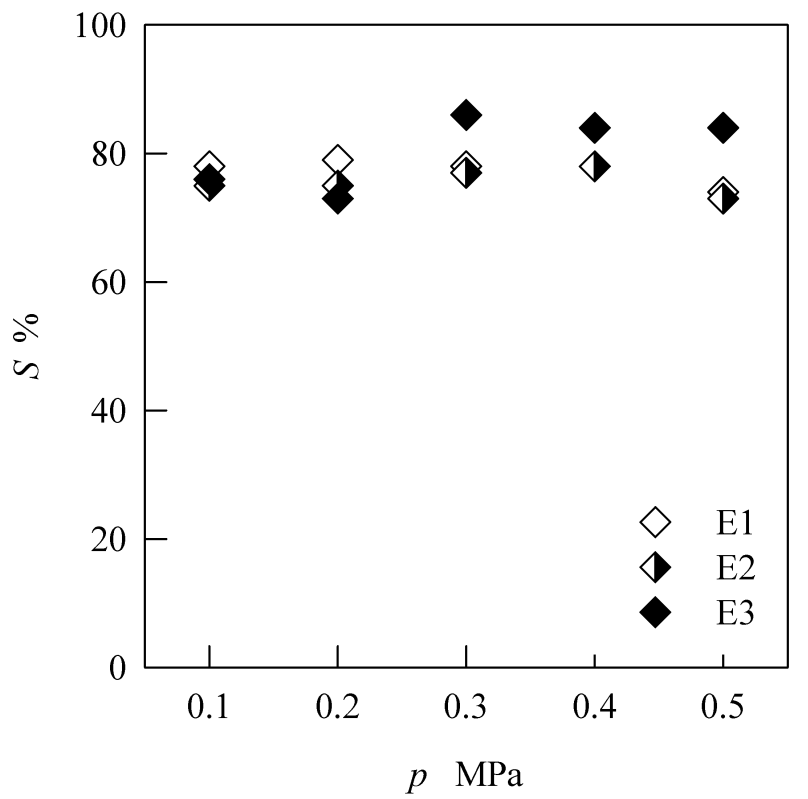

Figure 8. Comparison in S among E1, E2 and E3 at $F=2 \%$ and $v=$ $200 \mathrm{~m} / \mathrm{min}$. 


\section{Comparison between $\mathrm{T} 1$ and $\mathrm{T} 2$}

Figure 9 shows the comparison in $N$ between T1 and T2 at $v=$ 200,400 and $600 \mathrm{~m} / \mathrm{min}$ and $F=2 \%$.

From Figure 9, T2 produces the interlaced yarn with larger $N$ than T1. For T1, because of corner structures, under the action of compressed air, filaments cannot revolve along the wall of yarn channel continuously. Furthermore, filaments are easy to be blown to stay at corners and difficult to tangle with each other, so $N$ is smaller. As for T2, there are also corner structures. After compressed air hitting on the top of yarn channel, because the direction of airflow is downward, filaments are difficult to stay in corners $B$ and $C$ (Figure 2). At the position of corner $A$, because the force of airflow acting on the filaments is the strongest, filaments also cannot stay there for a long time.

Figure 10 shows the comparison in $S$ between T1 and T2 at $v$ $=200,400$ and $600 \mathrm{~m} / \mathrm{min}$ and $F=2 \%$.

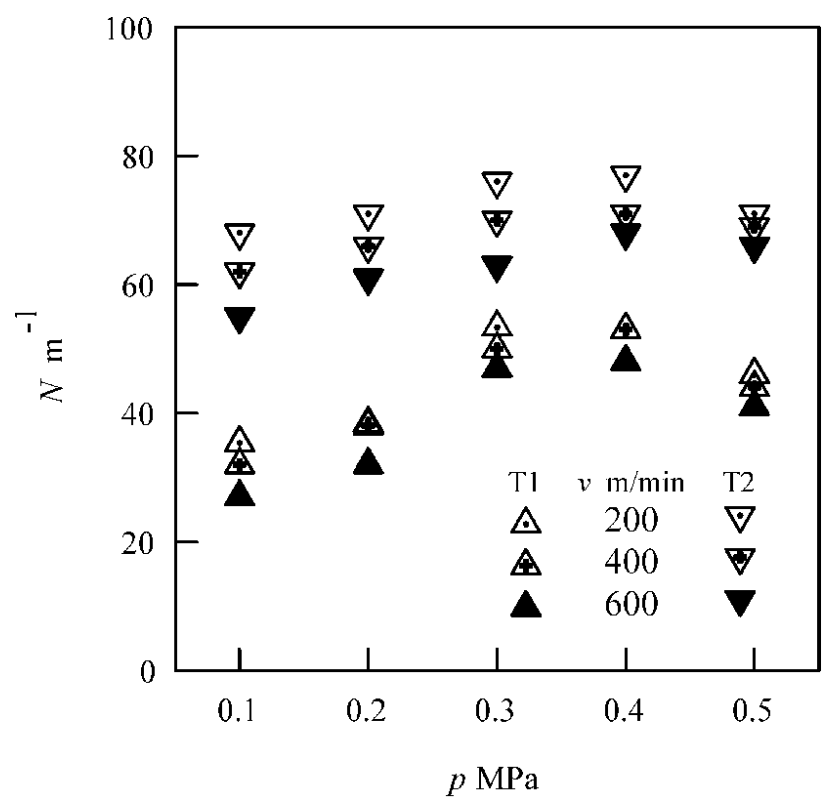

Figure 9. Comparison in $N$ between T1 and T2.

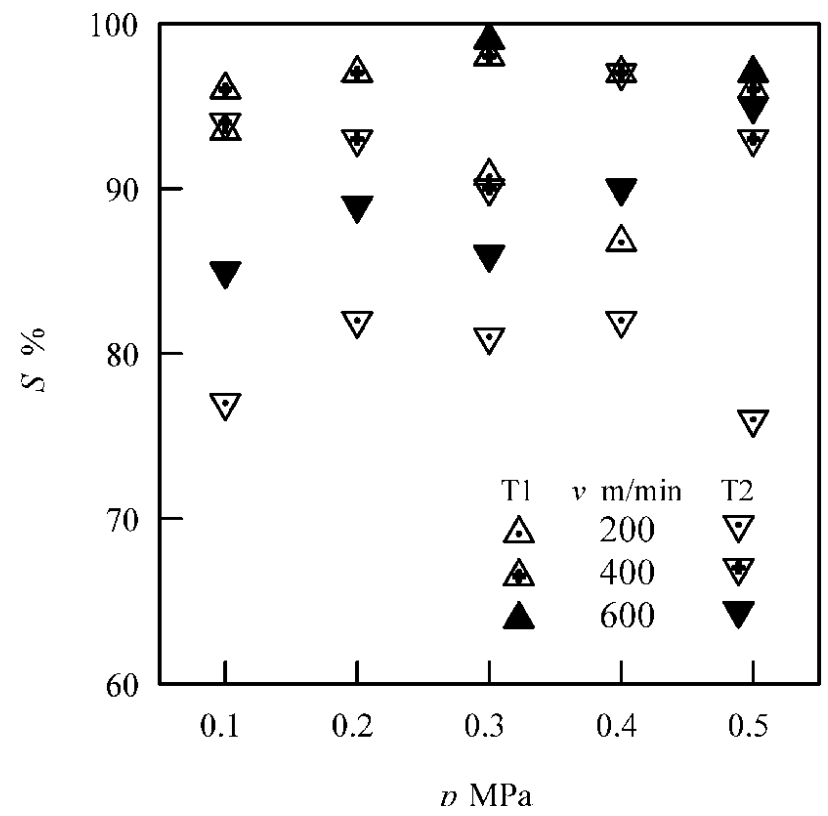

Figure 10. Comparison in $S$ between $\mathrm{T} 1$ and $\mathrm{T} 2$ at $F=2 \%$.
From Figure 10, T1 can produce interlaced yarn with higher $S$ than T2. For T1, acted by the force of the compressed air, filaments are easy to stay at position $A$ as shown in Figure 2 (T1). And because force of airflow is strong at position A, filaments can tangle with each other tightly.

\section{Comparison between T2 and E2}

Figure 11 shows the comparison in $N$ between T2 and E2 at $F$ $=2 \%$ and $v=200,400$ and $600 \mathrm{~m} / \mathrm{min}$. Figure 12 shows the comparison in $N$ between T2 and E2 at $p=0.4 \mathrm{MPa}$ and $F=$
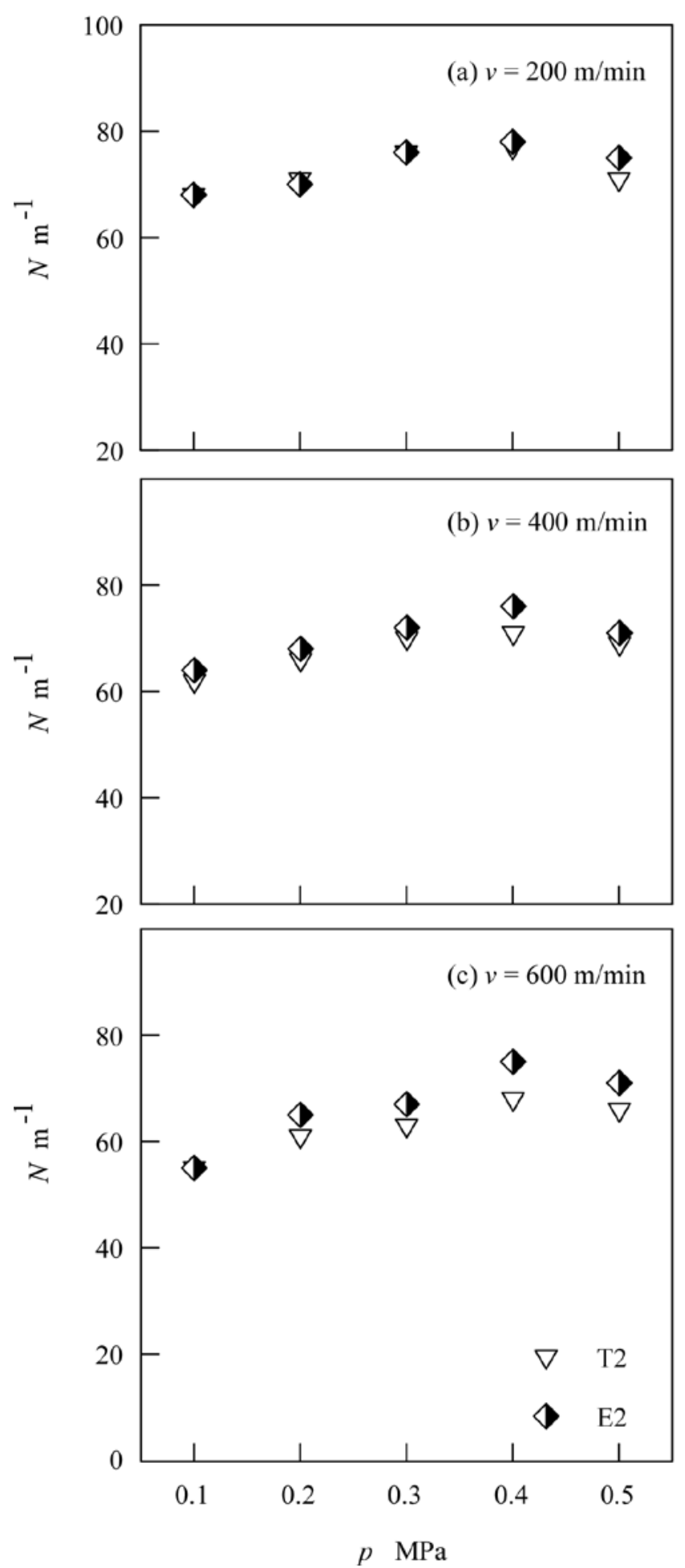

Figure 11. Comparison in $N$ between $\mathrm{T} 2$ and $\mathrm{E} 2$ at $F=2 \%$. 
$2 \%$. Figure 13 shows the comparison in $N$ between T2 and E2 at $p=0.4 \mathrm{MPa}$ and $v=200 \mathrm{~m} / \mathrm{min}$.

From Figures $11-13, N$ of the interlaced yarn produced with E2 is higher than that of T2. From the position of air jet nozzle, transverse length of yarn channel of E2 is longer than that of T2 in most positions of the ordinate. Larger space for filament opening and revolving is better for forming tangling parts.

Figure 14 shows the comparison in $S$ between T2 and E2 at $v$ $=200 \mathrm{~m} / \mathrm{min}$ and $F=2 \%$.

From Figure 14, we can see that T2 can produce the interlaced yarn with higher $S$ than E2. This is because a yarn in T2 will stay at the corners (positions $B$ and $C$ as shown in Figure 2) in the yarn channel for a longer time and be subjected to a relatively high speed airflow. On the other hand, since the yarn channel of E2 has round shape, the yarn can move more smoothly in the yarn channel and the relative velocity between air and yarn will be smaller.

\section{Conclusions}

In order to clarify the effects of cross-sectional shape of yarn channel on the performance of the interlacer, five interlacers with various yarn channel shape were designed. The performance of each interlace was evaluated from the number and strength of tangles of interlaced yarn it produced. These five interlacers were classified into two types: round shape, E1, E2 and E3, and cornered shape, T1 and T2. Among these interlacers, E2 and T2 are the better ones. The results obtained in this experiment are as follows:

- $\quad$ Cross-sectional shape of yarn duct affects both $N$ and $S$.

- When cross-sectional area of yarn duct is fixed, larger processing space is good for filament opening and then the amount of the tangles per unit length.

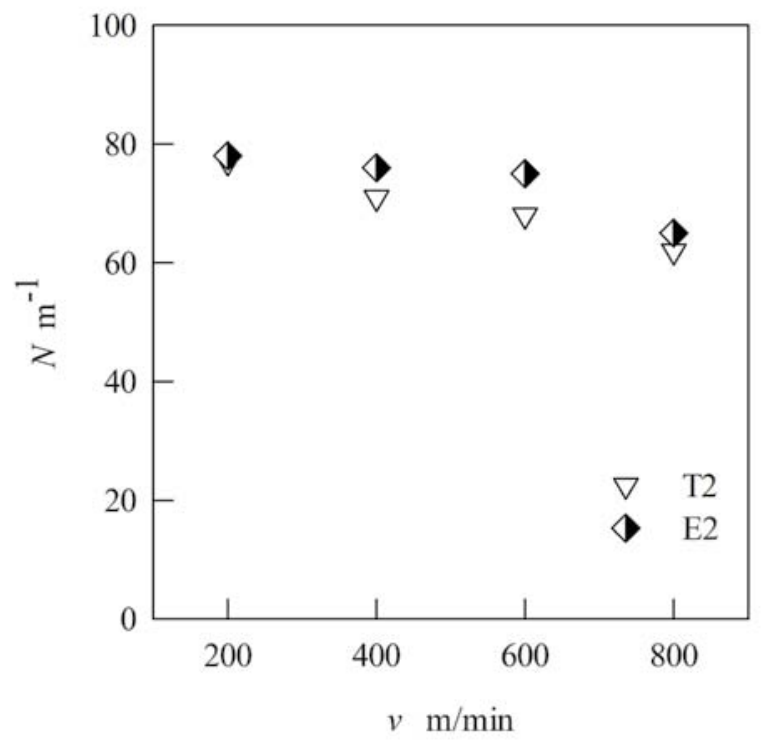

Figure 12. Comparison in $N$ between $\mathrm{T} 2$ and E2 at $p=0.4 \mathrm{MPa}$ and $F=2 \%$.
- Corner structures located at lower part (except at the position of air jet nozzle) of yarn duct are not good for interlacing.

- The interlacer with cross-sectional shape of yarn duct similar to a circle can produce interlaced yarn with larger $N$ and lower $S$.

\section{Acknowledgements}

The authors are grateful for the financial support by the Fundamental Research Funds for the Central Universities (No. JUSRP51301A).

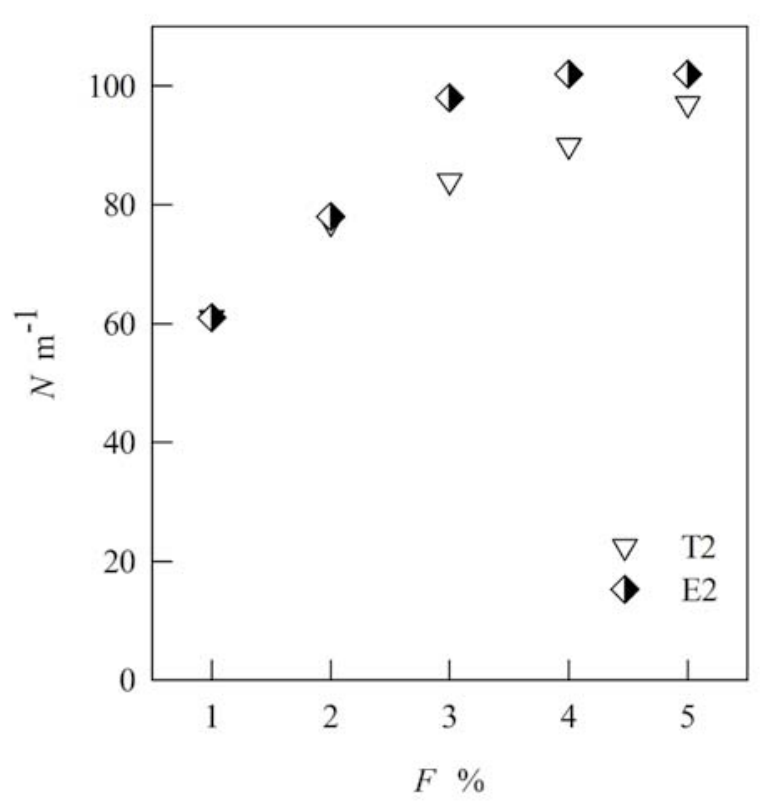

Figure 13. Comparison in $N$ between $\mathrm{T} 2$ and $\mathrm{E} 2$ at $p=0.4 \mathrm{MPa}$ and $v$ $=200 \mathrm{~m} / \mathrm{min}$.

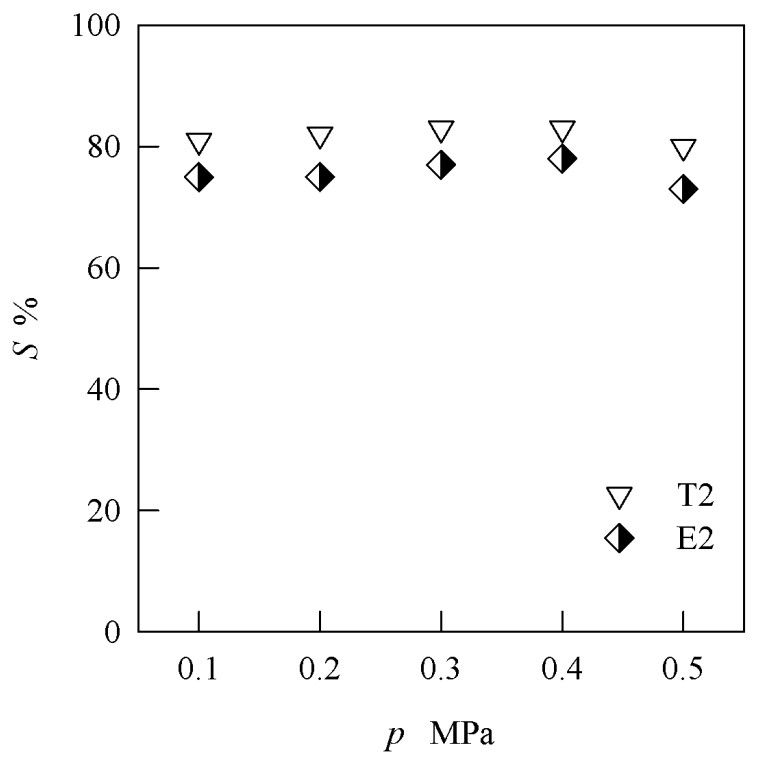

Figure 14. Comparison in $S$ between $\mathrm{T} 2$ and $\mathrm{E} 2$ at $v=200 \mathrm{~m} / \mathrm{min}$ and $F=2 \%$. 


\section{References}

[1] Dernir A. Intermingling/Interlacing-A survey[J]. Textile Asia. 1990, 21(9): 114-122.

[2] DuPont D N. Compact Interlaced Yarn[P]. 1961.5.

[3] lemoto Y, Chono S, Kasamatsu K. Size Effect of Interlacer Part1: Number of Tangles and Yarn Motion[J]. Journal of the Textile Machinery Society of Japan. 1999, 45(3): 71-77.

[4] Miao M, Song M C C. Air Interlaced Yarn Structure and Properties[J]. Textile Res. J. 1995, 65(8): 433-440.

[5] lemoto Y, Chono S, Tanaka T. Study on Interlaced Yarn Part 6:Yarn Motion in Interlacer[J]. Journal of the Textile Machinery Society of Japan. 1999, 45(2): 44-50.

[6] lemoto $Y$, Tanoue $S$, Lu J, et al. Effects of Size of Interlacer on Air Flow in a Yarn Duct.[J]. Journal of Textile Engineering. 2009, 55(4): 111-118.

[7] Yoshiyuki I, Shuichi $T$, Jun $L U$, et al. Effects of Size of Interlacer on Air Flow in a Yarn Duct.[J]. Journal of Textile Engineering. 2009, 55(4).
[8] Juraeva $M$, Song $D J$, Ryu K J. An optimum design study of the yarn-channel shape of the air-interlacing nozzle by analysis of fluid flow[J]. Textile Research Journal. 2012, 82(5): 474-483.

[9] Murakami K, Tokunaga K. Characteristics of compressible air flow in an interlacer and yarn entanglement[J]. Journal of Textile Engineering. 2006: 121-129.

[10]Versteeg $H K$, Acar M. Effect of Geometry on the Performance of Intermingling Nozzles[J]. Textile Res. J. 1999, 69(8): 545-551.

[11] Murakami K T. Characteristics of Incompressible Air Flow in an Interlacer[J]. Journal of Textile Engineering. 2006, 52(2): 73-79.

[12] Qiu H, lemoto Y, Tanoue S. Yarn Motion in Interlacers with Various Cross-sectional Shapes of Yarn Duct[J]. Journal of Textile Engineering. 2007, 53(2): 59-67. 